\title{
LIGHT STERILE NEUTRINOS IN COSMOLOGY
}

\author{
Stefano Gariazzo ${ }^{a}$ \\ Department of Physics, University of Torino, and INFN, Sezione di Torino, Via P. \\ Giuria 1, I-10125 Torino, Italy
}

\begin{abstract}
We briefly discuss the main effects of the presence of a Light Sterile Neutrino $(\mathrm{LS} \nu)$ in Cosmology and how its properties can be constrained by Cosmic Microwave Background (CMB) and other cosmological measurements.
\end{abstract}

\section{Introduction}

The measurements of neutrino oscillations in atmospheric, solar and longbaseline neutrino oscillation experiments firmly indicate that at least two neutrino mass eigenstates have a positive mass (see e.g. Refs. [1-3]). The mixing between the flavour neutrino eigenstates, that we indicate with $\nu_{\alpha}(\alpha=e, \mu, \tau)$, and the mass neutrino eigenstates, indicated with $\nu_{k}(k=1,2,3)$, can be written in terms of the mixing matrix $U_{\alpha k}$. The mixing relation is $\nu_{\alpha}=\sum U_{\alpha k} \nu_{k}$. The mixing matrix $U_{\alpha k}$ is described by three mixing angles, a CP violating phase and two Majorana phases, that are physical only if neutrino are Majorana particles. The description of the three neutrino mixing is completed by the existence of two squared-mass differences $\Delta m_{S O L}^{2}$ and $\Delta m_{A T M}^{2}$, which are measured in neutrino oscillation experiments (see e.g. Ref. [3]).

Among the numerous neutrino oscillation experiments, it is possible to find some anomalies that cannot be explained in the context of the three neutrino mixing. These anomalies are related to the results obtained in short-baseline (SBL) neutrino oscillation experiments by the LSND experiment [4], by several experiments measuring the electron antineutrino flux from nuclear reactors 5 and in the calibration of the GALLEX and SAGE Gallium solar neutrino experiments [6] (see Ref. 77 for a full list of references). A possible explanation of these anomalies involves new neutrino oscillations, driven by a third squaredmass difference $\Delta m_{S B L}^{2} \simeq 1 \mathrm{eV}$, that implies the existence of a fourth neutrino mass eigenstate. This additional light neutrino degree of freedom should be mainly sterile. We assume that the active neutrinos have a negligible mass with respect to this light sterile neutrino ( $\mathrm{LS} \nu$ ). The squared-mass difference $\Delta m_{S B L}^{2}$ implies that the sterile neutrino has a mass $m_{s} \simeq m_{4} \simeq \sqrt{\Delta m_{S B L}^{2}} \simeq$ $1 \mathrm{eV}$. The opposite choice, that is an inverted sterile hierarchy with $m_{4} \ll$ $m_{1}, m_{2}, m_{3}$, is forbidden by the cosmological bounds on the neutrino masses $[8$ and by the experimental bounds on neutrinoless double- $\beta$ decay, assuming that massive neutrinos are Majorana particles (see Ref. 9]).

\footnotetext{
${ }^{a}$ E-mail: gariazzo@to.infn.it
} 


\section{Neutrino Parameterization}

To describe the contribution of the LS $\nu$ in Cosmology we must separate the epoch when it was relativistic from the final part of the Universe evolution, when it is non-relativistic.

The contribution of all the relativistic species to the energy density of radiation $\rho_{r}$ in the early Universe can be written in terms of the effective number of relativistic species $N_{\text {eff }}$, defined through:

$$
\rho_{r}=\left[1+\frac{7}{8}\left(\frac{4}{11}\right)^{4 / 3} N_{\mathrm{eff}}\right] \rho_{\gamma},
$$

being $\rho_{\gamma}$ the energy density of photons. The contribution of the three active neutrinos is $N_{\mathrm{eff}}^{\mathrm{sm}}=3.046$, larger than 3 because of the non-instantaneous decoupling of the neutrinos from the electron-photon fluid and of the entropy transfer at the electron decoupling [10]. If the $\mathrm{LS} \nu$ is fully thermalized with the active neutrinos, its contribution to $N_{\text {eff }}$ should be 1 and $N_{\text {eff }}=4.046$. Assuming that only photons and neutrinos are relativistic in the early Universe, the contribution of the $\mathrm{LS} \nu$ can be written as 11

$$
\Delta N_{\mathrm{eff}}=N_{\mathrm{eff}}-N_{\mathrm{eff}}^{\mathrm{sm}}=\left[\frac{7}{8} \frac{\pi^{2}}{15} T_{\nu}{ }^{4}\right]^{-1} \frac{1}{\pi^{2}} \int d p p^{3} f_{s}(p),
$$

where $T_{\nu}$ is the active neutrino temperature, $p$ is the neutrino momentum and $f_{s}(p)$ is the $\mathrm{LS} \nu$ momentum distribution function.

Since the neutrino temperature today is $T_{\nu} \propto 10^{-4} \mathrm{eV}$, the $\mathrm{LS} \nu$ is no more relativistic. The energy density of the LS $\nu$ when it becomes non-relativistic can be parameterized through the effective mass $m_{s}^{\text {eff }}$, defined as [1] 12]:

$$
\frac{m_{s}^{\mathrm{eff}}}{94.1 \mathrm{eV}}=\frac{h^{2}}{\rho_{c}} \frac{m_{s}}{\pi^{2}} \int d p p^{2} f_{s}(p),
$$

where $h$ is the reduced Hubble parameter and $\rho_{c}$ is the critical energy density.

Both $\Delta N_{\text {eff }}$ and $m_{s}^{\text {eff }}$ depend on the LS $\nu$ momentum distribution function $f_{s}(p)$. If the $\mathrm{LS} \nu$ is relativistic at decoupling, $f_{s}(p)$ does not depend on its mass $m_{s}$. Since sterile and active neutrinos are mixed, and since the LS $\nu$ does not have electroweak interactions, its decoupling cannot occur later than that of the active neutrinos, that takes place at temperatures of about $1 \mathrm{MeV}$. Depending on the production mechanism of the $\mathrm{LS} \nu$ there are two main possibilities for $f_{s}(p)$. If the $\mathrm{LS} \nu$ is produced through some thermal (TH) process, its momentum distribution function can be written as a Fermi-Dirac function at a temperature $T_{s}$, that is $f_{s}^{T H}(p)=\left(e^{p / T_{s}}+1\right)^{-1}$. In this case it is possible to show that $m_{s}^{\mathrm{eff}}=\Delta N_{\mathrm{eff}}^{3 / 4} m_{s}$. If the production is non-thermal, instead, there 
are several possible scenarios. A popular one is the non-resonant production or Dodelson-Widrow (DW) scenario [13. In the DW scenario the $\mathrm{LS} \nu$ momentum distribution function can be written as $f_{s}^{D W}(p)=\beta\left(e^{p / T_{\nu}}+1\right)^{-1}$, where $\beta$ is a normalization factor, and one obtains $m_{s}^{\text {eff }}=\Delta N_{\text {eff }} m_{s}$.

\section{Neutrino Effects on the CMB spectrum}

The effective number of relativistic species controls the expansion rate $H$ in the early Universe and the time of the matter-radiation equality. This influences the CMB spectrum in several ways. The redshift of matter-radiation equality is defined by $1+z_{\mathrm{eq}}=\omega_{r} / \omega_{m}$, where $\omega_{i}=\rho_{i} h^{2} / \rho_{c}$ is the density parameter of the species $i$ and the subscript $m$ is for the matter component. If $N_{\text {eff }}$ is increased, the equality is delayed and $H$ at $\mathrm{CMB}$ decoupling is higher. The consequences are 7,14 an increase of the first peak of the CMB, due to the early Integrated Sachs-Wolfe (ISW) effect, and a shift at higher multipoles of the angular scale of the acoustic peaks. These effects can be seen comparing the black $\left(\Delta N_{\text {eff }}=0\right)$ and the red $\left(\Delta N_{\text {eff }}=2\right)$ spectra in Fig. 1. If one wants to restore the matter-radiation equality to the former redshift, it is necessary to rescale the matter density. In order to maintain the redshift of the matter- $\Lambda$ equality, where $\Lambda$ indicates the cosmological constant, it is necessary to vary also the $\Lambda$ energy density. If one varies together $\omega_{m}$ and $N_{\text {eff }}$ (blue), or $\omega_{m}, \omega_{\Lambda}$ and $N_{\text {eff }}$ (green), the effects of an higher $N_{\text {eff }}$ can be partially compensated. In the last case, the effect of an higher energy density at all the times produces a suppression of the CMB spectrum at high- $\ell$, also known as Silk damping effect.

As its mass is around $1 \mathrm{eV}$, the $\mathrm{LS} \nu$ is still relativistic at the time of matterradiation equality. As a consequence, its mass can influence only the late-time evolution. In particular, the impact of $m_{s}$ on the CMB spectrum is visible mainly through the slope of the CMB spectrum at low multipoles and through the effect of neutrino free-streaming. The slope of the CMB spectrum at low multipoles is related to the late ISW effect, originated after the Universe enters the $\Lambda$-dominated phase. Neutrino free-streaming occurs since they have a large velocity and they cannot cluster at small scales. After the non-relativistic transition, however, their velocity diminishes and they start to cluster, as dark matter does. The comoving free-streaming scale reaches a maximum at the time of the non-relativistic transition, that occurs at the wavemode $k_{\mathrm{nr}} \simeq$ $0.0178 \Omega_{m}^{1 / 2}\left(T_{\nu} / T_{s}\right)^{1 / 2}\left(m_{s} / 1 \mathrm{eV}\right)^{1 / 2} h \mathrm{Mpc}^{-1}$. All the wavemodes below $k_{\mathrm{nr}}$ are not affected by the neutrino free-streaming, while all the wavemodes $k \gtrsim k_{\mathrm{nr}}$ are suppressed. The amount of the suppression depends on the neutrino masses, but it is not particularly strong in the CMB spectrum. The best way to measure the neutrino masses in Cosmology, in fact, is to study the free-streaming effect in the power spectrum of matter perturbations. Future experiments such as DESI 15$]$ and Euclid [16] will put strong constraints on the neutrino masses, 


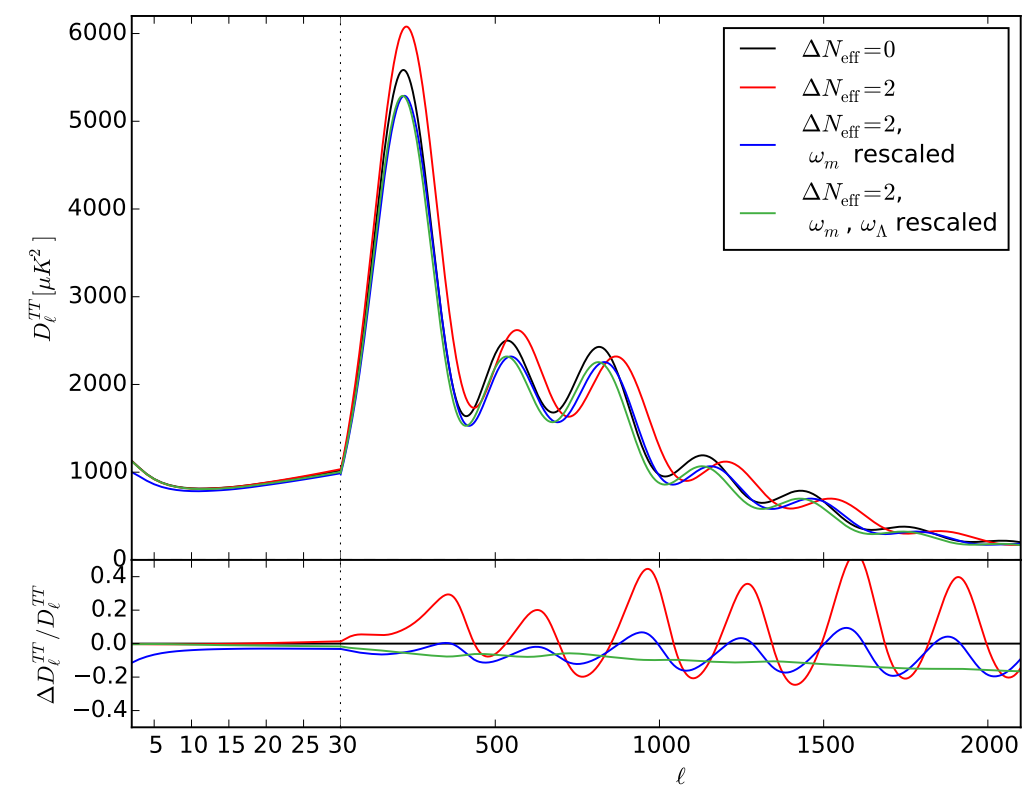

Figure 1: Comparison of different CMB temperature spectra predicted by the theoretical model. We show the effects of altering $N_{\text {eff }}$ alone or in combination with $\omega_{m}$ and $\omega_{\Lambda}$.

and possibly on the neutrino mass hierarchy 17,18 .

\section{Current Constraints from Cosmology}

Several joint analyses of cosmological and SBL neutrino oscillation data have been performed in the past. Here we present the results obtained in Ref. [19, where the joint analyses are based on the CMB temperature spectrum from the Planck 2013 release [20], on the low- $\ell$ polarization spectra from WMAP 21] and on the high- $\ell$ spectrum calculated through the ACT/SPT likelihood $[22]$.

To derive constraints on the $\mathrm{LS} \nu$ properties we consider an extension of the well-known $\Lambda$ CDM model, expanded with the $\mathrm{LS} \nu$ presented in the previous Sections. The constraints are obtained from the cosmological datasets alone or adding the results of the analysis of SBL neutrino oscillations data as a prior for the LS $\nu$ mass. In the left panel of Fig. 2 we show the marginalized constraints in the $m_{s}^{\text {eff }}-N_{\text {eff }}$ plane obtained from the analysis of the CMB data mentioned above, both without SBL prior (black) or with a SBL prior for the mass of a DW (red) or a TH (blue) LS $\nu$. Dotted (dashed) lines indicate constant masses, with values written next to each line, for the DW (TH) model. As we can 

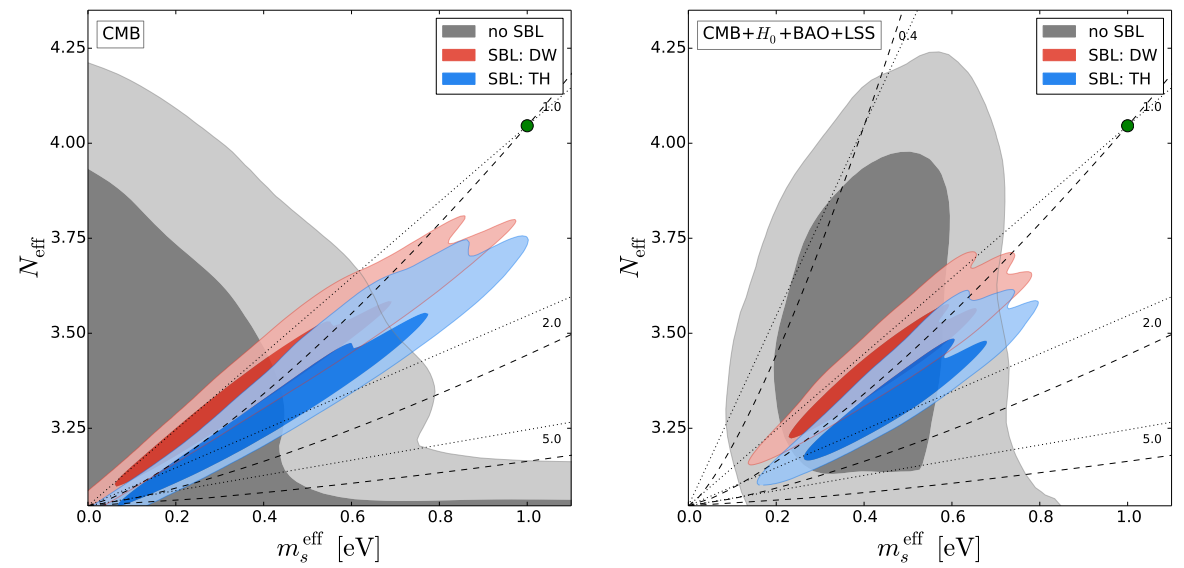

Figure 2: Constraints at $68 \%$ and $95 \%$ CL in the $m_{s}^{\text {eff }}-N_{\text {eff }}$ plane from the CMB data alone (left panel) and from a combination of $\mathrm{CMB}+H_{0}+\mathrm{BAO}+\mathrm{LSS}$ data (right panel). The green point indicates a fully thermalized $\mathrm{LS} \nu$ with $m_{s}=1 \mathrm{eV}$. Dotted (dashed) lines indicate constant masses, for values written in the figures, for the DW (TH) model. From 7 .

see, the SBL prior forces the physical mass to lie in the range allowed by SBL experiments, with the consequence that not all the values of $m_{s}^{\text {eff }}-N_{\text {eff }}$ favoured by the CMB data are compatible with the SBL data. A fully thermalized LS $\nu$ is allowed only if it has a very small mass, but for the SBL masses an incomplete thermalization is required, being $N_{\text {eff }} \lesssim 3.75$ at $95 \%$ CL. Larger LS $\nu$ masses are allowed only if $N_{\text {eff }}$ is close to 3.046 .

The $\Lambda$ CDM model explains the majority of the cosmological observations, but some tensions exist. In particular, in the context of the $\Lambda$ CDM model a tension between the determinations of the matter fluctuations and of the Hubble parameter at high- and low-redshift may appear, possibly because of unaccounted systematics. The value of $H_{0}$ determined by Planck 8, 12 is in tension with the local determinations, that give higher values 23.24. At the same time, the amount of matter fluctuations at small scales, encoded in the parameter $\sigma_{8}$, is smaller if obtained from local measurements of weak lensing 25 or from cluster counts 26] than when derived from CMB constraints 8,12 .

A LS $\nu$ has the potential to reconcile local and cosmological observations. This is the consequence of the correlation between $N_{\text {eff }}$ and $H_{0}$, and of the LS $\nu$ free-streaming, that reduces the matter fluctuations at small scales. In the right panel of Fig. 2 we show the bounds for the $\mathrm{LS} \nu$ as derived from an extended dataset, that includes the same $\mathrm{CMB}$ data as above, plus the measurements of the Baryon Acoustic Oscillations (BAO), a prior on $H_{0}$ and one on $\sigma_{8}$ from Large Scale Structures (LSS). As we anticipated, the fit prefers positive LS $\nu$ masses in order to reduce the predicted value of $\sigma_{8}$ through the neutrino free- 
streaming, and $N_{\text {eff }}>3.046$ in order to have an higher $H_{0}$. Even in this case, however, the full thermalization of the LS $\nu$ is strongly disfavoured.

\section{Open Problems and Conclusions}

Despite the fact that the LS $\nu$ can explain some of the tensions between the current cosmological observations at high- and low-redshift, it seems that a full reconciliation is rather complicated. The main reason is that we do not have a valid explanation to the fact that the $\mathrm{LS} \nu$ is not fully thermalized. With the mixing parameters determined by SBL oscillations, in fact, the LS $\nu$ should fully thermalize with the active neutrinos 27. Moreover, it seems that the LS $\nu$ cannot fully reconcile both the $H_{0}$ and the $\sigma_{8}$ tensions, since the direction of the degeneracies in the $\sigma_{8}-H_{0}$ plane is not optimal [8].

Unless a new mechanism that explains the low thermalization of the $\mathrm{LS} \nu$ is found (see e.g. Ref. 7 for some possibilities), the current status of the global analyses shows that the SBL neutrino seems to be excluded by Cosmology. Future investigations of SBL neutrino oscillations and of Cosmology are required to grow a more complete knowledge of the theories and to explain of the present tensions between the different experimental probes.

\section{Acknowledgments}

The work of S. G. is supported by the Theoretical Astroparticle Physics research Grant No. 2012CPPYP7 under the Program PRIN 2012 funded by the Ministero dell'Istruzione, Università e della Ricerca (MIUR).

[1] Carlo Giunti and Chung W. Kim. Fundamentals of Neutrino Physics and Astrophysics. 2007.

[2] Samoil Bilenky. Introduction to the physics of massive and mixed neutrinos, volume 817. Springer, 2010.

[3] K.A. Olive et al. Review of particle physics. Chin.Phys., C38:090001, 2014.

[4] A. Aguilar-Arevalo et al. Evidence for neutrino oscillations from the observation of anti-neutrino(electron) appearance in a anti-neutrino(muon) beam. Phys. Rev., D64:112007, 2001.

[5] G. Mention et al. The Reactor Antineutrino Anomaly. Phys. Rev., D83:073006, 2011.

[6] Carlo Giunti and Marco Laveder. Short-Baseline Active-Sterile Neutrino Oscillations? Mod. Phys. Lett., A22:2499-2509, 2007.

[7] S. Gariazzo et al. Light sterile neutrinos. arXiv:1507.08204 [hep-ph].

[8] P. A. R. Ade et al. Planck 2015 results. XIII. Cosmological parameters. arXiv:1502.01589 [astro-ph.CO]. 
[9] S. M. Bilenky and C. Giunti. Neutrinoless Double-Beta Decay: a Probe of Physics Beyond the Standard Model. Int. J. Mod. Phys., A30:1530001, November 2015.

[10] Gianpiero Mangano et al. Relic neutrino decoupling including flavor oscillations. Nucl. Phys., B729:221-234, 2005.

[11] Mario A. Acero and Julien Lesgourgues. Cosmological constraints on a light non-thermal sterile neutrino. Phys. Rev., D79:045026, 2009.

[12] P. A. R. Ade et al. Planck 2013 results. XVI. Cosmological parameters. Astron. Astrophys., 571:A16, November 2014.

[13] Scott Dodelson and Lawrence M. Widrow. Sterile-neutrinos as dark matter. Phys. Rev. Lett., 72:17-20, 1994.

[14] Maria Archidiacono et al. Cosmic dark radiation and neutrinos. Adv. High Energy Phys., 2013:191047, 2013.

[15] Michael Levi et al. The DESI Experiment, a whitepaper for Snowmass 2013. arXiv:1308.0847 [astro-ph.CO].

[16] R. Scaramella et al. Euclid space mission: a cosmological challenge for the next 15 years. IAU Symp., 306:375-378, 2015.

[17] Andreu Font-Ribera et al. DESI and other dark energy experiments in the era of neutrino mass measurements. JCAP, 1405:023, 2014.

[18] Benjamin Audren et al. Neutrino masses and cosmological parameters from a Euclid-like survey: Markov Chain Monte Carlo forecasts including theoretical errors. JCAP, 1301:026, 2013.

[19] S. Gariazzo et al. Light Sterile Neutrinos in Cosmology and ShortBaseline Oscillation Experiments. JHEP, 11:211, 2013.

[20] P. A. R. Ade et al. Planck 2013 results. I. Overview of products and scientific results. Astron. Astrophys., 571:A1, November 2014.

[21] C. L. Bennett et al. Nine-Year Wilkinson Microwave Anisotropy Probe (WMAP) Observations: Final Maps and Results. Astrophys. J. Suppl., 208:20, 2013.

[22] J. Dunkley et al. The Atacama Cosmology Telescope: likelihood for small-scale CMB data. JCAP, 1307:025, 2013.

[23] Adam G. Riess et al. A 3\% Solution: Determination of the Hubble Constant with the Hubble Space Telescope and Wide Field Camera 3. Astrophys. J., 730:119, 2011.

[24] George Efstathiou. H0 Revisited. Mon. Not. Roy. Astron. Soc., 440:1138-1152, 2014.

[25] Catherine Heymans et al. CFHTLenS: The Canada-France-Hawaii Telescope Lensing Survey. Mon. Not. Roy. Astron. Soc., 427:146, 2012.

[26] P. A. R. Ade et al. Planck 2013 results. XX. Cosmology from SunyaevZeldovich cluster counts. Astron. Astrophys., 571:A20, 2014.

[27] Steen Hannestad et al. Thermalisation of light sterile neutrinos in the early universe. JCAP, 1207:025, 2012. 